\title{
DETERMINING THE WORKSPACE IN CASE OF THE ROBOTS WITH PARALLEL STRUCTURE DELTA 3DOF
}

\author{
ANDRIOAIA, D[ragos]; PASCU, M[arius]; MIHAILA, L[ucian] \& OBREA, C[laudiu] F[lorin]
}

\begin{abstract}
Within the last years, the robots with parallel structure Delta 3DOF are used more and more in applications that require: transporting objects at high speed, transporting heavy objects, at a high accuracy. Because the parallel structures have a limited workspace, researches on the workspace amplified lately. This work is approaching the study of determining the workspace for a parallel structure Delta 3DOF. The method being used allows the numerical determining of the workspace shape, as well as its limits. At the same time, this method helps with establishing the methods for optimizing the workspace so that the largest possible workspace is provided.
\end{abstract}

Keywords: workspace, parallel structure Delta 3DOF, workspace simulation

\section{INTRODUCTION}

Within the last years, the robots that include a parallel structure attracted the attention of the academic world's researchers. Among the most famous robots with parallel structure are those provided with the parallel structure Delta 3DOF. By comparing the robots that include serial structure manipulators to those including parallel structure manipulators, it may be noticed that: the parallel structure has a series of advantages, such as high rigidity, availability for transporting heavier objects, more accurate positioning [1]. The disadvantages are: narrower workspace, more difficult control [1].

The workspace of the robots has been intensively studied along the years by several researchers. The most common workspaces, as per Merlet, are: the translation workspace, the orientation workspace, the accessible workspace, the inclusive orientation workspace, the total orientation workspace, dexterity workspace, total workspace with reduced orientation [1] and [2]. Kumar introduces the denomination of controllable dexterity workspace [2].

The motion of the robot manipulators within the working space may be restricted by several factors, such as: the constructive limits of the passive kinematical couplings, the limits given by the driving devices of the active kinematical couplings, cohesions given by the constructive elements of the robot as well as by points or areas of singularity that may divide the workspace into various component parts [1]. An important parameter to be considered during the study of the robot workspace is the number of freedom degrees that the mobile platform has. The parallel structure Delta 3DOF (three parallel mechanisms), was invented at the beginning of the decade 1980 by the professor Reymond Clavel [3].
Fig. 1 shows the CAD model of the parallel structure Delta 3DOF. The parallel structure Delta 3DOF is composed of: one fixed platform - 1, one mobile platform -2 , to which the element to be handled is fixed -9 . The fixed platform is interconnected with the mobile platform by means of three kinematical linkages. From the constructive point of view, the kinematical linkages are identical, but functionally, they differ. Each kinematical linkage is actuated through a driving system - 3. The driving system conveys the motion to the leading element -5 , through a kinematic rotation coupling -4 ; the leading - 5 conveys the motion to the parallel elements being led - 7 through the spherical kinematic couplings -6 . The parallel elements being led -7 convey the motion to the mobile platform - 2 by means of the kinematic couplings -8 .

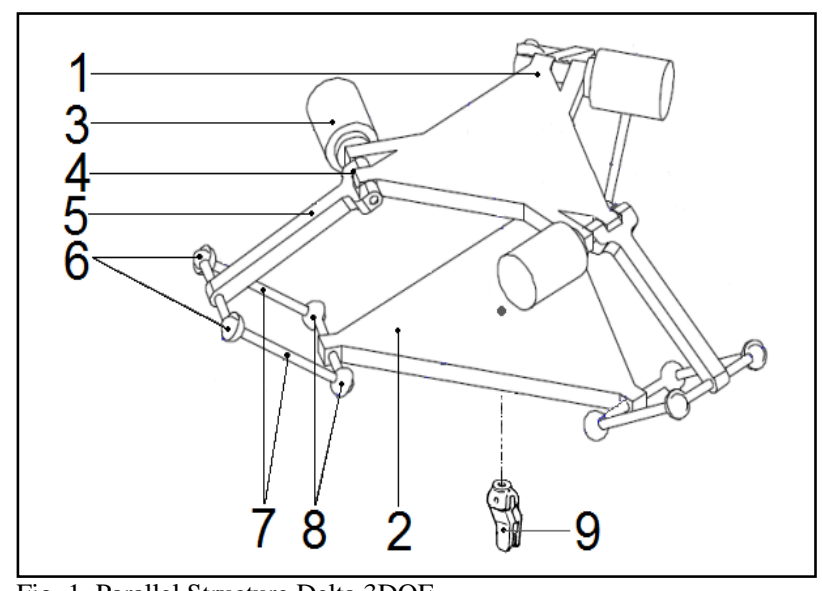

Fig. 1. Parallel Structure Delta 3DOF

A characteristic of this type of robot would be the fact that, at the variation of the angles between the joints, various positioning of the handled object can be obtained in the workspace.

By considering the importance of the workspace in this work, the authors approach the study for determining the workspace accessible for a robot with parallel structure Delta 3DOF. The work is structured as follows:

The first chapter presents the theoretical aspects on the workspace as well as the presentation of the parallel structure being analysed. Chapter 2 presents the geometrical parameters that compose the parallel structure of type Delta 3DOF. Chapter 3 presents the reverse kinematical model of the parallel structure Delta 3DOF. The workspace of the parallel structure Delta 3DOF will be determined by using the reverse geometrical model of the structure. Chapter 4 presents 
the method used for determining the workspace. At Chapter 5 the experimental results obtained further to the implementation of the respective method using the Matlab working environment are presented. Chapter 6 presents the conclusions resulting after the experimental analyses.

\section{DETERMINING THE GEOMETRICAL PARAMETERS}

Fig. 2 below presents the parameterized kinematic diagram of one kinematic linkage of the parallel structure of type Delta 3DOF.

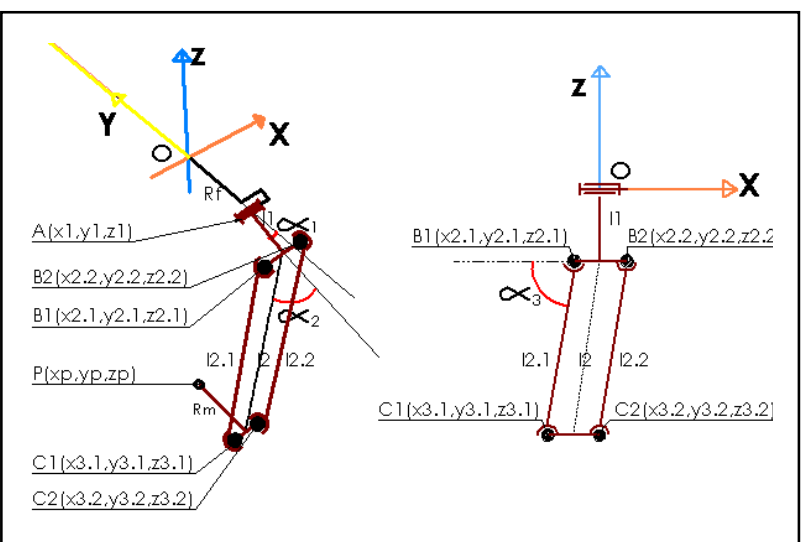

Fig. 2. Kinematic diagram of one kinematic linkage of the parallel structure Delta 3DOF

Starting from the kinematic diagram of one kinematic linkage shown at Fig. 2, the geometrical diagram of a parallel structure Delta 3DOF has been issued, as shown at Fig. 3. In the geometrical diagram the parallel elements -7 , Fig. 1 have been modelled through a single element, by taking into consideration their functionality within the structure.

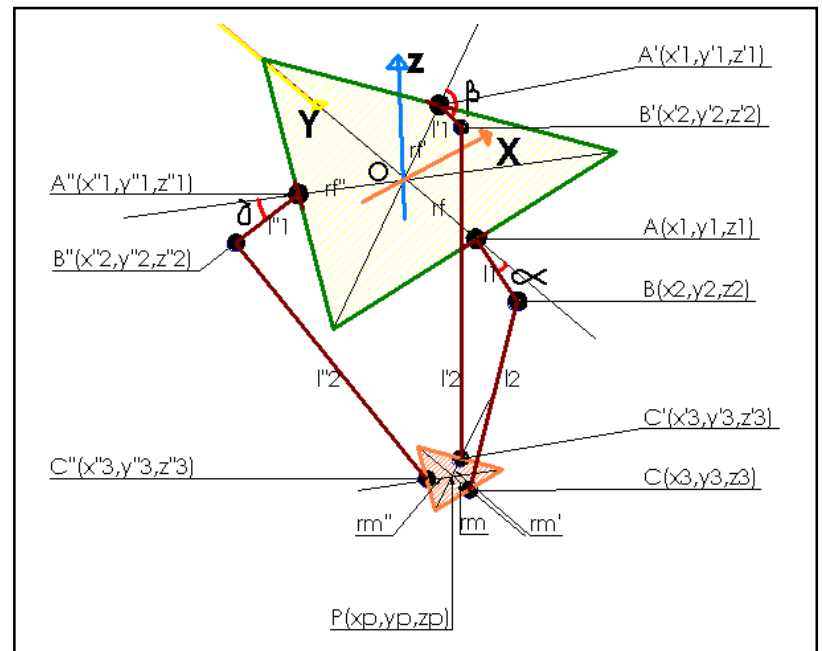

Fig. 3. Geometrical diagram of the parallel structure Delta 3DOF

The three kinematical linkages are located at each $120^{\circ}$ on the two platforms. In Fig. 3 it may be noticed how the three kinematical linkages are located on the fixed platform. In order to simplify the mathematical model, the first kinematical linkage has been oriented to the same direction as the $\mathrm{Y}$ axis of the fixed reference system.

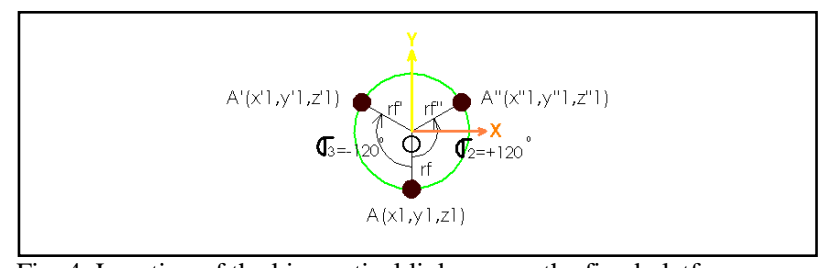

Fig. 4. Location of the kinematical linkages on the fixed platform

The geometrical parameters and identities used within the structure are presented in Tab. 1:

\begin{tabular}{|l|l|}
\hline Geometrical & $r f, r f^{\prime}, r f^{\prime \prime}, l_{1}, l_{1}^{\prime}, l_{1}^{\prime \prime}, l_{2}, l_{2}^{\prime}, l_{2}^{\prime \prime}, r m$, \\
parameters & $r m^{\prime}, r m^{\prime \prime}, \alpha, \beta, \gamma, \sigma_{1}, \sigma_{2}, \sigma_{3} ;$ \\
\hline Geometrical identities & $r f=r f^{\prime}=r f^{\prime \prime}$ - Fixed platform radius; \\
& $l_{1}=l_{1}^{\prime}=l_{1}^{\prime \prime}$ - Length of the leading element; \\
& $l_{2}=l_{2}^{\prime}=l_{2}^{\prime \prime}$ - Length of the led element; \\
& $r m=r m^{\prime}=r m^{\prime \prime}$ - Mobile platform radius. \\
\hline
\end{tabular}

Tab. 1. Geometrical parameters and identities being used

\section{REVERSE KINEMATICAL PROBLEM}

For the reverse kinematical model the angular values $\alpha, \beta, \gamma$ will be determined in function of the coordinates of the object to be handled $\mathrm{P}\left(x_{p}, y_{p}, z_{p}\right)$ [4]. By using the reverse kinematical model of the work [5], the solutions of the reverse kinematical model are presented at (1), (9), (17).

$$
\begin{gathered}
\alpha=2\left[\tan ^{-1}\left(\frac{-M_{1} \pm \sqrt{\mathrm{M}_{1}^{2}+J_{1}^{2}-K_{1}^{2}}}{\left(K_{1}-J_{1}\right)}\right)\right] \\
J_{1}=2 l_{1}\left(y_{3} \cos \left(\sigma_{1}\right)+x_{3} \sin \left(\sigma_{1}\right)-r f\right) \\
\mathrm{K}_{1}=L_{1}-2 r f\left(y_{3} \cos \left(\sigma_{1}\right)+x_{3} \sin \left(\sigma_{1}\right)\right) \\
L_{1}=l_{2}^{2}-l_{1}^{2}-y_{3}^{2}-x_{3}^{2}-z_{3}^{2} \\
M_{1}=2 l_{1} z_{3} \\
x_{3}=x_{p}+r m \sin \left(\sigma_{1}\right) \\
y_{3}=y_{p}+r m \cos \left(\sigma_{1}\right) \\
z_{3}=z_{p}
\end{gathered}
$$

By considering that the first kinematical linkage has the same orientation as the $\mathrm{Y}$ axis of the reference system, it will result $\sigma_{1}=0$.

$$
\begin{gathered}
\beta=2\left[\tan ^{-1}\left(\frac{-M_{2} \pm \sqrt{\mathrm{M}_{2}^{2}+J_{2}^{2}-K_{2}^{2}}}{\left(K_{2}-J_{2}\right)}\right)\right] \\
J_{2}=2 l_{3}^{\prime}\left(y_{3}^{\prime} \cos \left(\sigma_{2}\right)+x_{3}^{\prime} \sin \left(\sigma_{2}\right)-r_{f}^{\prime}\right) \\
K_{2}=L_{2}-2 r_{f}^{\prime}\left(y_{3}^{\prime} \cos \left(\sigma_{2}\right)+x_{3}^{\prime} \sin \left(\sigma_{2}\right)\right) \\
L_{2}=l_{2}^{\prime 2}-l_{1}^{\prime 2}-y_{3}^{\prime 2}-x_{3}^{\prime 2}-z_{3}^{\prime 2} \\
M_{2}=2 l_{1}^{\prime} z_{3}^{\prime 2} \\
x_{3}^{\prime}=x_{p}+r m^{\prime} \sin \left(\sigma_{2}\right) \\
y_{3}^{\prime}=y_{p}+r m^{\prime} \cos \left(\sigma_{2}\right) \\
z_{3}^{\prime}=z_{p}
\end{gathered}
$$


Where: $\sigma_{2}=120^{\circ}$.

$$
\begin{gathered}
\vee=2\left[\tan ^{-1}\left(\frac{-M_{3} \pm \sqrt{\mathrm{M}_{3}^{2}+J_{3}^{2}-K_{3}^{2}}}{\left(K_{3}-J_{3}\right)}\right)\right] \\
J_{3}=2 l_{1}^{\prime \prime}\left(y_{3}^{\prime \prime} \cos \left(\sigma_{3}\right)+x_{3}^{\prime \prime} \sin \left(\sigma_{3}\right)-r_{f}^{\prime \prime}\right) \\
K_{3}=L_{3}-2 r_{f}^{\prime \prime}\left(y_{3}^{\prime \prime} \cos \left(\sigma_{3}\right)+x_{3}^{\prime \prime} 2 \sin \left(\sigma_{3}\right)\right) \\
L_{3}=l_{2}^{\prime \prime 2}-l_{1}^{\prime \prime} 2-y_{3}^{\prime \prime 2}-x_{3}^{\prime \prime 2}-z_{1}^{\prime \prime} 2 \\
M_{3}=2 l_{1}^{\prime \prime} z_{3}^{\prime \prime} 2 \\
x_{3}^{\prime \prime}=x_{p}+r m^{\prime \prime} \sin \left(\sigma_{3}\right) \\
y_{3}^{\prime \prime}=y_{p}+r m^{\prime \prime} \cos \left(\sigma_{3}\right) \\
z_{3}^{\prime \prime}=z_{p}
\end{gathered}
$$

Where: $\sigma_{3}=240^{\circ}$.

The variables: $J_{1}, K_{1}, L_{1}, M_{3}, J_{2}, K_{2}, L_{2}, M_{3}, J_{3}, K_{3}$, $L_{3}, M_{3}$ have been used for solving the reverse kinematical problem.

\section{DESCRIPTION OF THE METHOD USED FOR DETERMINING THE WORKSPACE}

The workspace will be determined through a method similar to the methods presented in the papers [1], [2], [6], [7]. The method has a numerical solution. The diagram by which the workspace will be determined is presented in Fig. 5.

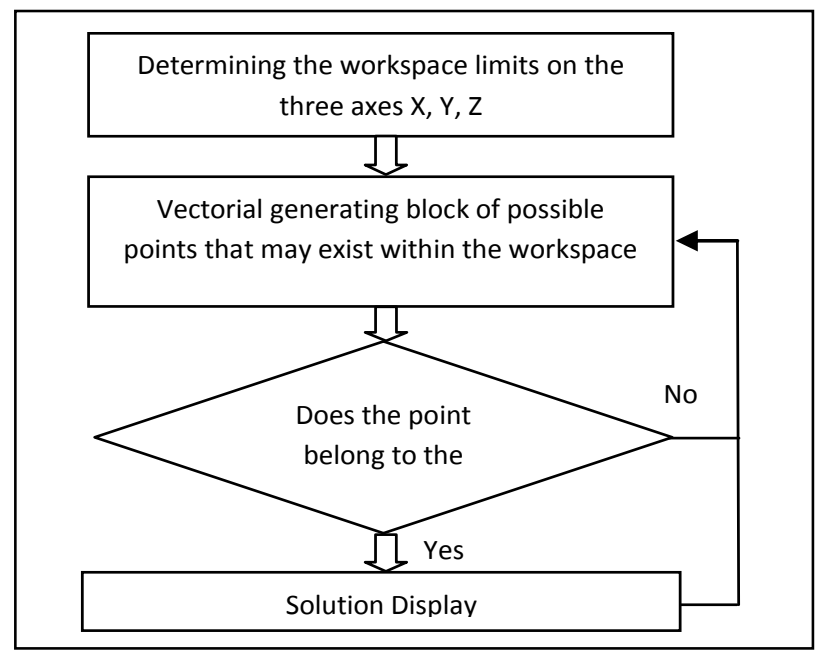

Fig. 5. Block diagram used for determining the workspace

The workspace is determined by going through the following stages:

Step1. By means of the block for determining the workspace limits the points of maximum will be established: $x_{\max }, y_{\max }, z_{\max }$, and minimum $x_{\min }, y_{\min }$, $Z_{\min }$ that the handled object may reach on the three axes $(\mathrm{X}, \mathrm{Y}, \mathrm{Z})$ of the fixed reference system [1]. The limits of the workspace can be approximated by using the following relations:

$$
\begin{gathered}
x_{\max } \cong y_{\max }=l_{2}+r m-r f \\
x_{\min } \cong y_{\min }=-\left(l_{2}+r m-r f\right)
\end{gathered}
$$

$$
\begin{gathered}
z_{\text {min }}=0 \\
z_{\text {max }}=l_{1}+l_{2}
\end{gathered}
$$

From (25), (26), (27) and (28) it results that the maximum and minimum values of the coordinates of the points that the handled object $\mathrm{P}\left(x_{p}, y_{p}, z_{p}\right)$ can reach are included to the following intervals: (29), (30), (31).

$$
\begin{aligned}
& x_{p} \in\left[\begin{array}{lll}
x_{\min } & \ldots & x_{\max }
\end{array}\right] \\
& y_{p} \in\left[\begin{array}{lll}
y_{\min } & \ldots y_{\max }
\end{array}\right] \\
& z_{p} \in\left[\begin{array}{lll}
z_{\min } & \cdots & z_{\max }
\end{array}\right]
\end{aligned}
$$

The workspace limits previously determined will give the limits of a cube, as shown in Fig. 6, inside which the workspace of the parallel structure Delta $3 \mathrm{DOF}$ is inscribed [1].

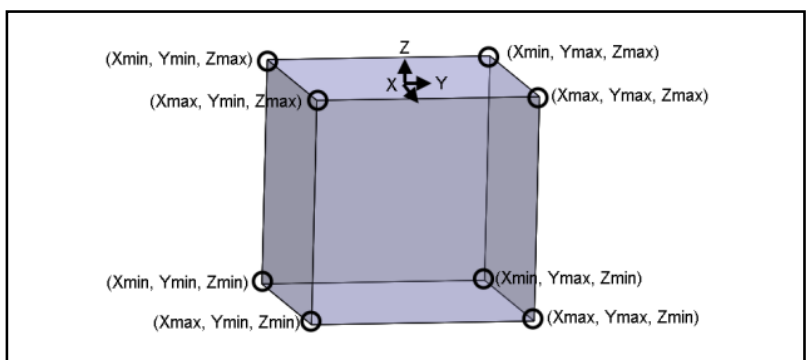

Fig. 6. Representation of the limits of a cube, having the workspace of a parallel structure Delta 3DOF inscribed inside this cube

Step 2. With the help of the vector generating block of possible points that may exist inside of the workspace, a three-dimensional network of points inside the cube will be generated. The generation of the points inside the cube is performed by digitizing the three intervals of the workspace limits through a digitizing element $a$ (32), (33), (34), [1], [7].

$$
\begin{gathered}
x_{p}=-x_{\max }: a: x_{\max } \\
y_{p}=-y_{\max }: a: y_{\max } \\
z_{p}=-z_{\max }: a: 0
\end{gathered}
$$

By combining the values of the digitizing performed on the workspace limits the coordinates of the points generated within the network will be obtained, as shown in Fig. 7.

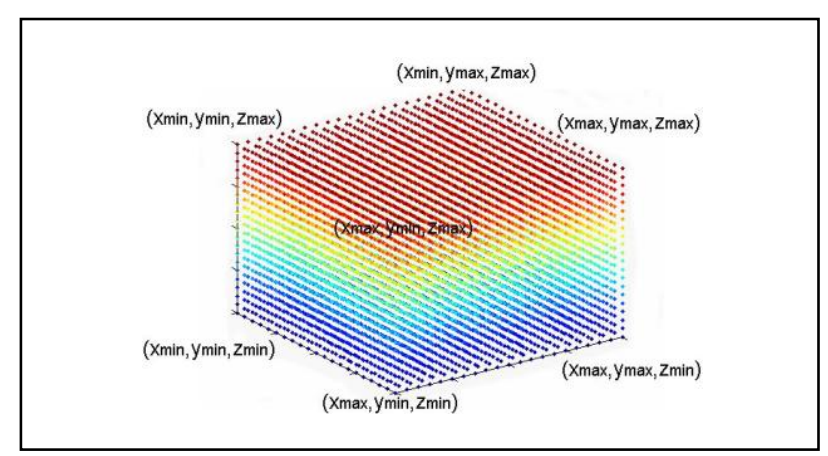

Fig. 7. Generation of the points possibly existing within the workspace

The generated points will have the values of the coordinates included to the workspace limits previously 
calculated. The maximum number of points $n_{p}$ that can be generated inside the cube volume is given by (35):

$$
n_{p}=\left(\frac{\left|-x_{\max }\right|+\left|x_{\max }\right|}{a}\right)\left(\frac{\left|-y_{\max }\right|+\left|y_{\max }\right|}{a}\right)\left(\frac{\left|-z_{\max }\right|+\left|z_{\max }\right|}{a}\right)
$$

Step 3. The coordinates of each generated point will be verified with the help of the block that verifies whether the point is located inside the workspace. In the reverse geometrical model of the parallel structure Delta $3 \mathrm{DOF}$ the coordinates of the generated points will be replaced and then it will be verified whether the following conditions are complied with: the reverse geometrical model has a solution; the solution of the reverse geometrical model belongs to the restricted intervals of the kinematic mover couplings as well as to other constraints that may restrict the manipulator motion, discussed at Chapter 1 [1], [2]. If the above conditions are met, the analysed point will belong to the workspace of the parallel structure Delta 3DOF [1].

Step 4. If the point is located inside the workspace, it will be displayed [1]. The algorithm finishes at the moment when all points possibly to exist inside the workspace have been generated and verified.

\section{EXPERIMENTAL RESULTS}

For simulating the workspace a parallel structure has been proposed, featuring the constructive dimensions and restrictions shown in Tab. 2:

\begin{tabular}{|c|c|}
\hline Constructive dimensions & $\begin{array}{c}\text { Values of the constructive } \\
\text { dimensions }\end{array}$ \\
\hline$r f=r f^{\prime}=r f^{\prime \prime}$ & 170 \\
\hline$r m=r m^{\prime}=r m^{\prime \prime}$ & 70 \\
\hline$l_{1}=l_{1}^{\prime}=l_{1}^{\prime \prime}$ & 40 \\
\hline$l_{2}=l_{2}^{\prime}=l_{2}^{\prime \prime}$ & 150 \\
\hline$\alpha_{\max }=\beta_{\max }=\gamma_{\max }$ & $90^{0}$ \\
\hline$\alpha_{\min }=\beta_{\min }=\gamma_{\min }$ & $0^{0}$ \\
\hline
\end{tabular}

Tab. 2. Constructive dimensions and restrictions of the parallel structure Delta 3DOF

Where $\gamma_{\text {max }}, \beta_{\text {max }}, \alpha_{\max }$ are the maximum values of the limits of the active kinematic couplings, $\gamma_{\text {min }}, \beta_{\text {min }}$, $\alpha_{\min }$ are the minimum values of the limits of the live kinematic couplings. The digitizing increment of the intervals is $a=5$.

Further to the stimulation of the workspace in Matlab, the workspace of the parallel structure Delta 3 DOF, as shown in Fig. 8 and Fig. 9 has been obtained.

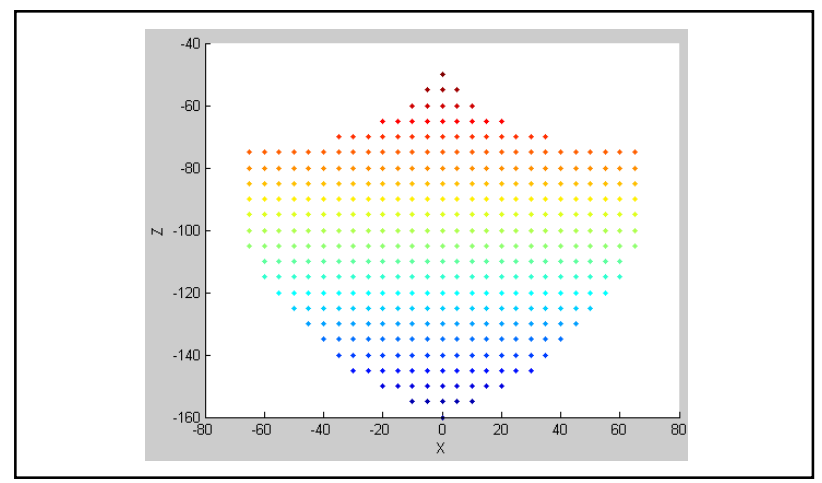

Fig. 8. Workspace of the parallel structure Delta 3DOF - view from the plane XZ

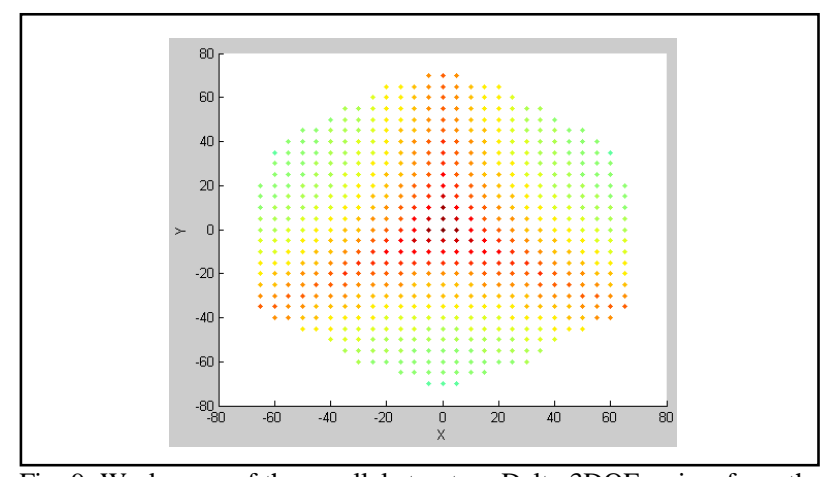

Fig. 9. Workspace of the parallel structure Delta 3DOF - view from the plane XY

All results presented at this section are obtained by an Intel Core i3 processor of $3.6 \mathrm{GHz}$, the programs are elaborated in compliance with MATLAB R2009b. The calculation time needed for obtaining the optimal solution is estimated at approximately 5 minutes.

\section{CONCLUSIONS}

By performing approximations closer to the real values of the workspace limits on the three axes, it will result a shortening of the calculation time of the program where the method of calculation is implemented. By giving as low as possible values for the digitizing increment $a$, more precise forms of the workspace will be obtained but this will lead to increasing the calculation time of the program.

Further to the simulation of the method it may be noticed that the workspace is located at the intersection of three tore. The shape of the workspace may differ in function of the constructive dimensions used on the parallel structure Delta 3DOF.

\section{REFERENCES}

[1] Laribi, M. A.; Romdhane, L. \& Zeghloul, S. (2008). Advanced Synthesis of the DELTA Parallel Robot for a Specified Workspace, In: Parallel Manipulators Towards New Applications, Huapeng, Wu, InTech, pp. 207-210, I-Tech Education and Publishing, ISBN: 978-3-902613-40-0, Rijeka

[2] Affi, Z.; Romdhane, L. \& Maalej, A. (2004). Dimensional synthesis of a 3-translational-DOF in-parallel manipulator for a desired workspace. European Journal of Mechanics - A/Solids, Vol 23, No. 2, 2008, pp. 311-324, ISSN: 0997-7538

[3] Clavel, R. (1990). Device for movement and displacing of an element in space, U.S. Patent, No. 4976582

[4] López, M.; Castillo, E.; García, G. \& Bashir, A. (2006). Delta robot: inverse, direct, and intermediate Jacobians. Journal ofMechanical Engineering Science, Vol. 220, No. C1, 2006, pp.103-109, ISSN: 09544062

[5] Traslosheros, A.; Angel, L.; Sebastián, J. M.; Roberti, F.; Carelli, R. \& Vaca R. (2010). New visual Servoing control strategies in tracking tasks using a PKM, Available from: http:// www.intechopen.com/books/mechatronic-systems-simulationmodeling-and-control/new-visual-servoing-control-strategies-intracking-tasks-using-a-pkm, Accessed: 2012-04-21

[6] Liu, X.-J.; Wang, J.; Oh, K.-K. \& Kim, J. (2004). A New Approach to the Design of a DELTA Robot with a Desired Workspace, Journal of intelligent \& robotics systems, Vol. 39, No. 2, 2004, pp. 209-225, ISSN: 0921-0296

[7] Liu, X.-J.; Wang, J. \&Zheng, H. (2003). Workspace atlases for the computer aided design of the Delta robot, Journal of Mechanical Engineering Science, Vol. 217, No. 8, 2006, pp. 861869, ISSN: 09544062 